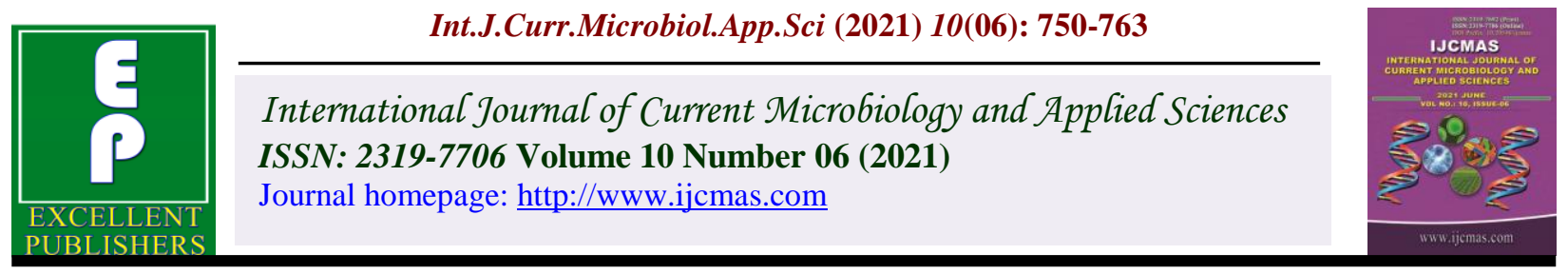

Original Research Article https://doi.org/10.20546/ijcmas.2021.1006.082

\title{
Effect of INM on Content of NPKS Zn in Grain and Stover of Maize-Wheat Cropping System
}

\author{
Pushpendra Kumar*, S. D. Dubey, U. S. Tiwari, R. K. Pandey, \\ Karam Hussain and R. K. Singh
}

Department of Soil Science and Agril. Chemistry, C. S. Azad University of Agriculture and Technology, Kanpur-208002, India

*Corresponding author

Keywords

Vermicompost,

Farm yard manure, recommended dose of nitrogen

Article Info

Accepted: 25 May 2021 Available Online: 10 June 2021

A field experiment was conducted at Student's Instructional Farm of Chandra Shekhar Azad University of Agriculture and Technology, Kanpur to study the effect of Vermicompost, FYM, Sulphur, Zinc, Azotobacter and PSB on growth, yield and uptake of nutrients in maize and their residual effect on succeeding wheat was studied during 2018-19 and 2019-20. A significant improvement was recorded with respect to content of nutrients in grain as well as stover/straw of maize and succeeding wheat with the application of S and Zn @ 20 and $5 \mathrm{~kg} \mathrm{ha}^{-1}$ along with RDF of NPK as compared to control during both the years. The maximum content of nutrients (NPKSZn) were estimated with the application of $\mathrm{T}_{14}(100 \%$ $\mathrm{RDN}+25 \% \mathrm{~N}-\mathrm{VC}+\mathrm{S}+\mathrm{Zn}+\mathrm{Az}+\mathrm{PSB}$ ) but remained statistically at par with the treatments of $\mathrm{T}_{13}$ where FYM was given instead of Vermicompost in maize and wheat during both the years of experimentation.

\section{Introduction}

Maize (Zea mays), is known as "queen of cereals", the world's third most important cereal crop in term of production stands after wheat and rice. The maize contributes 9 per cent of total food grain production of the country. The area under maize cultivation is $8.6 \mathrm{mha}$ with a production $21.7 \mathrm{mt}$. in India whereas, in U.P. the production is 1.5 ton/ha with an area 1.1 mha (Anonymous, 2013). It is cultivated with different crop-sequence under various agro-climatic regions of the country. Hence, it is considered as potential driver of crop diversification under different situations (Jat et al., 2011).

Wheat is the most important cereal crop of the world. It is the staple food of different countries of the world. With the existing ricewheat system there is emerging challenges of natural resource degradation, declining crop 
productivity and ecological problems. Among different maize-base cropping systems, maizewheat cropping system ranks first (Jat et al., 2011).

The application of well decomposed farm Yard Manure(FYM) to soil has been practiced for many centuries for increasing crop yield, soil organic matter, microbial activities and improving soil fertility and soil structure for sustainable agriculture for long time (Blair et al., 2005; Kundu et al., 2006) however, proper combination of both organic and inorganic fertilizers have better effects on crop growth and development and yield component of crop than alone (Budaruddin et al., 1999: Hossain et al., 2002: Manna et al., 2005). Balance application of $\mathrm{N}, \mathrm{P}, \mathrm{K}$ fertilizers with FYM was best option for higher crop yield in maize wheat cropping system (Brar, et al., 2015).

The inoculation of biofertilizer in combination with limited doses of rock phosphate or SSP produced higher and sustainable crop yield, maintained the soil health and reduced the $\mathrm{P}$ fertilizer cost (Singhal et al., 2012). The excess and imbalance use of nutrients allow continuous nutrient mining from the soil resulting to poor the crop productivity and soil health. Integrated nutrient management system may be suitable for sustainable yield, quality traits and better environment. The existing nutrient management practices are based mainly on individual crop.

There is need to develop integrated nutrient management module in a cropping system. There is meager information about integrated nutrient management on maize-wheat cropping system. Therefore, with keeping in mind the present scenario the experiment was carried out to evaluate the effect of integration of organic and inorganic sources along bioinoculants on growth, yield and uptake of nutrients in maize and their residual effect on wheat.

\section{Materials and Methods}

A field experiment was conducted during kharif and rabi seasons of 2018-19 and 201920 at Student's Instructional Farm, C.S. Azad University of Agriculture and Technology, Kanpur (UP) situated at between $25^{0} 26^{\prime}$ to 26 ${ }^{0} 58$ ' North latitude and $79^{0} 31$ ' to $80{ }^{0} 34$ ' East longitude at an elevation of $125.9 \mathrm{~m}$ above mean sea level.

The region falls under agro-climatic zone $\mathrm{V}$ (Central Plain Zone) of Uttar Pradesh. The soil of the experimental field was alluvial in origin. The experiment was laid out in completely randomized block design with three replications treatments replicated thrice and plot size was $6 \times 5 \mathrm{~cm}^{2}$. Maize Variety Azad Uttam was sown in kharif whereas HD2967 was taken as wheat variety during rabi season. was $\mathrm{g}$ In the present experiment fourteen treatments viz., $\mathrm{T}_{1}$ (control), $\mathrm{T}_{2}(75 \%$ RDN), $\mathrm{T}_{3}(75 \% \mathrm{RDN}+25 \% \mathrm{~N}-\mathrm{FYM}), \mathrm{T}_{4}(75$ $\% \mathrm{RDN}+25 \% \mathrm{~N}-\mathrm{VC}), \mathrm{T}_{5}(75 \% \mathrm{RDN}+25 \%$ $\mathrm{N}-\mathrm{FYM}+\mathrm{S}+\mathrm{Zn}+\mathrm{Az}+\mathrm{PSB}), \mathrm{T}_{6}(75 \% \mathrm{RDN}$ $+25 \% \quad \mathrm{~N}-\mathrm{VC}+\mathrm{S}+\mathrm{Zn}+\mathrm{Az}+\mathrm{PSB}), \mathrm{T}_{7}(100 \%$ $\mathrm{RDN}), \mathrm{T}_{8}(100 \% \mathrm{RDN}+\mathrm{S}), \mathrm{T}_{9}(100 \% \mathrm{RDN}+$ $\mathrm{S}+\mathrm{Zn}), \mathrm{T}_{10}(100 \% \mathrm{RDN}+\mathrm{S}+\mathrm{Zn}+\mathrm{Az}+\mathrm{PSB}), \mathrm{T}_{11}$ $(100 \% \quad \mathrm{RDN}+25 \quad \% \quad \mathrm{~N}-\mathrm{FYM}), \quad \mathrm{T}_{12} \quad(100 \%$ $\mathrm{RDN}+25 \% \mathrm{~N}-\mathrm{VC}), \mathrm{T}_{13}(100 \% \mathrm{RDN}+25 \% \mathrm{~N}-$ $\mathrm{FYM}+\mathrm{S}+\mathrm{Zn}+\mathrm{Az}+\mathrm{PSB}), \mathrm{T}_{14}(100 \% \mathrm{RDN}+25$ $\% \mathrm{~N}-\mathrm{FYM}+\mathrm{S}+\mathrm{Zn}+\mathrm{Az}+\mathrm{PSB})$ were applied in maize.

Whereas in wheat a similar RDF (@ 120:60:40) of NPK was given in all the treatments of maize. The soil of the experimental field was sandy loam in texture which was low in organic carbon $\left(3.35 \mathrm{~g} \mathrm{~kg}^{-1}\right)$, slightly alkaline in reaction. Furthermore, the soil was low in available $\mathrm{N}\left(156 \mathrm{~kg} \mathrm{ha}^{-1}\right)$, medium in available $\mathrm{P}\left(10.34 \mathrm{~kg} \mathrm{ha}^{-1}\right)$, high in available $\mathrm{K}$ (198.16 $\left.\mathrm{kg} \mathrm{ha}^{-1}\right)$, low in available $\mathrm{S}\left(14.20 \mathrm{~kg} \mathrm{ha}^{-1}\right.$ and medium in available $\mathrm{Zn}$ (0.36 mg kg-1). Treatment wise plant samples were collected from each plot at harvest of the 
crop for analysis of N, P, K, S and Zn content and their uptake in grain and Stover/straw of Maize and wheat.

The five plants having intact leaves were selected randomly from each plot. The stover/straw samples were first air-dried and kept in oven at $60-70{ }^{0} \mathrm{C}$ for drying till the 12 hours to become free from moisture. Afterwards the samples were ground in a willey mill and stored in clean polythene bags. Similarly, dried grain samples were also ground oven dried, passed through 2 mesh sieve and stored in the sample bottles.

\section{Determination of $\mathrm{N}, \mathrm{P}, \mathrm{K}, \mathrm{S}$ and $\mathrm{Zn}$ content} in plant samples

\section{Nitrogen}

$\mathrm{N}$ is determined by Kjeldahl method given by Jackson (1967).

\section{Phosphorus}

$\mathrm{P}$ is determined colorimetrically by vanadatemolybdate yellow colour method as advocated by Chapman and Pratt (1961).

\section{Potassium}

$\mathrm{K}$ determination has been done using flame photometric method (Chapman and Pratt, 1961) outlined by Jackson (1967).

\section{Sulphur}

$\mathrm{S}$ is determined through turbidimetric method (Chesnin and Yien, 1956).

\section{Zinc}

$\mathrm{Zn}$ is extracted from plant with the help of atomic absorption spectrophotometer (Lindasey and Norwell, 1978).

\section{Results and Discussion}

\section{Effect of treatments on content of nutrients}

Nitrogen content in grain and stover of maize

The concentration of $\mathrm{N}$ in grain and stover showed significant variation due to treatments during both the years as well as on pooled mean basis as presented in table 1.0. The addition of either FYM or VC besides $75 \%$ or $100 \%$ RDN increased the $\mathrm{N}$ content in seed and stover. However, the addition of $\mathrm{S}+\mathrm{Zn}$, Azotobacter +PSB with $75 \%$ RDN+FYM or $\mathrm{VC}$ significantly contributed for $\mathrm{N}$ content in grain and stover. The highest $\mathrm{N}$ content were recorded under $\mathrm{T}_{14}(100 \% \mathrm{RDN}+25 \% \mathrm{~N}$ $\mathrm{VC}+\mathrm{S}+\mathrm{Zn}+\mathrm{Az}+\mathrm{PSB})$ which had at par effect with $\mathrm{T}_{13} \quad(100 \% \quad \mathrm{RDN}+25 \% \quad \mathrm{~N}-\mathrm{FYM}+\mathrm{S}$ $+\mathrm{Zn}+\mathrm{Az}+\mathrm{PSB}), \mathrm{T}_{10}(100 \% \mathrm{RDN}+\mathrm{S}+\mathrm{Zn}+\mathrm{Az}+$ $\mathrm{PSB})$ and $\mathrm{T}_{6}(75 \% \mathrm{RDN}+25 \% \mathrm{~N}-\mathrm{VC}+\mathrm{S}+\mathrm{Zn}+$ $\mathrm{Az}+\mathrm{PSB}$ ) but significantly superior to rest of the treatments. This may be due to organic sources considerably participates in mineralization of nutrients and easily absorbed by the plants and result to higher accumulation of nutrients. Similar results were also reported by Steinbach et al., (2004). These results are in agreement with the findings of (Rasool et al., 2016; Balyan and Kumpawat 2008). This might be due to the fact that combined application of organic and inorganic sources of nutrients modified the soil environment, besides providing the physical properties of soil and also the slow microbial decomposition of humus gradually increases the $\mathrm{N}$ availability during cropping period, which was manifested in higher $\mathrm{N}$ content maize.

\section{Phosphorus content}

It is evident from finding that increasing doses of $\mathrm{N}$ from 75 to $100 \%$ had increased the $\mathrm{P}$ content in grain and stover of maize. It was 
noticed that $\mathrm{P}$ content in grain as well as stover were higher in treatments consisting organic manure along with $75 \%$ and $100 \%$ RDN. The highest $\mathrm{P}$ content was recorded with the application of $\mathrm{T}_{14}(100 \% \mathrm{RDN}+25 \%$ $\mathrm{N}-\mathrm{VC}+\mathrm{S}+\mathrm{Zn}+\mathrm{Az}+\mathrm{PSB})$ however, statistically at par with the treatments of $\mathrm{T}_{13}(100 \%$ $\mathrm{RDN}+25 \% \quad \mathrm{~N}-\mathrm{FYM}+\mathrm{S}+\mathrm{Zn}+\mathrm{Az}+\mathrm{PSB}), \quad \mathrm{T}_{10}$ $(100 \% \mathrm{RDN}+\mathrm{S}+\mathrm{Zn}+\mathrm{Az}+\mathrm{PSB})$ and $\mathrm{T}_{6}(75 \%$ $\mathrm{RDN}+25 \% \mathrm{~N}-\mathrm{VC}+\mathrm{S}+\mathrm{Zn}+\mathrm{Az}+\mathrm{PSB})$ during both the years. The availability of phosphorus in soil may be increased due to use of organics as decomposition of organic manure progressed; various organic acids produced which solubilize phosphatase and other phosphate bearing minerals and thereby lower the phosphate fixation and higher absorption of P by plants Vidyavathi et al., (2012). Wilkinson et al., (1999) reported that $\mathrm{N}$ can increase $\mathrm{P}$ content in plants by increasing root growth, by increasing the ability of roots to absorb and translocates $\mathrm{P}$, and by decreasing soil $\mathrm{pH}$ as a result of absorption of $\mathrm{N}$. These results are in accordance with the findings of (Rayees et al., 2017 and Karki et al., 2005).

\section{Potassium content in maize}

The application of $100 \% \quad \mathrm{RDN}+25 \% \quad \mathrm{~N}-$ $\mathrm{VC}+\mathrm{S}+\mathrm{Zn}+\mathrm{Az}+\mathrm{PSB}$ ) recorded significantly higher $\mathrm{K}$ content than other treatments of 75 or $100 \%$ RDN integrated either with FYM or VC with or without S, Zn, Azotobacter and PSB but was statistically at par with the treatments $\mathrm{T}_{13}(100 \% \mathrm{RDN}+25 \% \mathrm{~N}-\mathrm{FYM}+$ $\mathrm{S}+\mathrm{Zn}+\mathrm{Az}+\mathrm{PSB}), \mathrm{T}_{10}(75 \% \mathrm{RDN}+\mathrm{S}+\mathrm{Zn}+\mathrm{Az}+$ PSB), $\mathrm{T}_{6}(75 \% \mathrm{RDN}+25 \% \mathrm{~N}-\mathrm{VC}+\mathrm{S}+\mathrm{Zn}+\mathrm{Az}+$ $\mathrm{PSB})$ and $\mathrm{T}_{5}(75 \% \mathrm{RDN}+25 \% \mathrm{~N}-\mathrm{FYM}+\mathrm{S}+$ $\mathrm{Zn}+\mathrm{Az}+\mathrm{PSB}$ ) however numerically superior. The results are in close conformity with the findings of (Brar et al., 2001 and Wagh 2002). It may be ascribed that the application of organic manure may have the solubilizing action of certain organic acids produced during their decomposition and hold $\mathrm{K}$ in available form thereby increasing its absorption and uptake (Vidyavathi et al., 2012).

\section{Sulfur content}

The application of $\mathrm{S}$ alone or in combination of $\mathrm{Zn}$ or $\mathrm{Zn}+\mathrm{Az}+\mathrm{PSB}\left(\mathrm{T}_{10}\right)$ along with $100 \%$ RDN increased the content of $S$ in grain and stover. Further combination of $\mathrm{S}$ with either VC or FYM increased the concentration of $S$ in grain and stover. The application of $\mathrm{T}_{14}$ $(100 \% \quad \mathrm{RDN}+25 \% \quad \mathrm{~N}-\mathrm{VC}+\mathrm{S}+\mathrm{Zn}+\mathrm{Az}+\mathrm{PSB})$ registered highest $\mathrm{S}$ concentration, however remained statistically at par with $\mathrm{T}_{13}(100 \%$ $\mathrm{RDN}+25 \% \quad \mathrm{~N}-\mathrm{FYM}+\mathrm{S}+\mathrm{Zn}+\mathrm{Az}+\mathrm{PSB}), \quad \mathrm{T}_{10}$ $(100 \% \mathrm{RDN}+\mathrm{S}+\mathrm{Zn}+\mathrm{Az}+\mathrm{PSB})$ and $\mathrm{T}_{6}(75 \%$ $\mathrm{RDN}+25 \% \quad \mathrm{~N}-\mathrm{VC}+\mathrm{S}+\mathrm{Zn}+\mathrm{Az}+\mathrm{PSB})$ but numerically higher to $\mathrm{T}_{1}$ (control).

The highest accumulation of S might be due to fortification of sulphur along with $100 \%$ NPK Joshi et al., (2013). Conjoint application of S with organic manure significantly increased the $\mathrm{S}$ uptake in grain and stover might be due to the synergistic action of Nitrogen and Sulfur dose. As organic manure enriches soil fertility through addition of organic matter in the soil along with sulfur application which enhances easy and faster mineralization of organic matter, which helps in increasing the availability and uptake of nutrient (Patel et al., (2003).

\section{Zn content}

The application of $\mathrm{Zn}$ significantly contributed for its concentration in grain and stover over no application. The highest $\mathrm{Zn}$ content was recorded under the treatment of $\mathrm{T}_{14}(100 \%$ $\mathrm{RDN}+25 \% \quad \mathrm{~N}-\mathrm{VC}+\mathrm{S}+\mathrm{Zn}+\mathrm{Az}+\mathrm{PSB})$ which was statistically at par with $\mathrm{T}_{13}(100 \%$ $\mathrm{RDN}+25 \% \quad \mathrm{~N}-\mathrm{FYM}+\mathrm{S}+\mathrm{Zn}+\mathrm{Az}+\mathrm{PSB}), \quad \mathrm{T}_{10}$ $(100 \% \mathrm{RDN}+\mathrm{S}+\mathrm{Zn}+\mathrm{Az}+\mathrm{PSB})$ and $\mathrm{T}_{6}(75 \%$ $\mathrm{RDN}+25 \% \quad \mathrm{~N}-\mathrm{VC}+\mathrm{S}+\mathrm{Zn}+\mathrm{Az}+\mathrm{PSB})$ and significantly superior to rest of the treatments.

The application of $\mathrm{Zn}$ increased its 
concentration in maize and it is further increased when applied in combination of $\mathrm{FYM}$ or $\mathrm{VC}+\mathrm{S}+\mathrm{Zn}+\mathrm{Az}+\mathrm{PSB})$. Increased content of zinc in grain and stover with integrated use of organic and inorganic fertilizers may be attributed due to the adequate supply of this nutrient thereby improved $\mathrm{Zn}$ content in grain and stover coupled with total biomass (Meena et al., 2006). Furthermore, it might be due to $\mathrm{Zn}$ involved in formation of chelates with organic ligands which lowered susceptibility to adsorption, fixation and precipitation in the soil ultimately improved absorption by plants during entire growth period (Kuniyal et al., 2012).

Effect on content of nutrients in grain and straw of wheat

\section{Nitrogen content}

The data regarding $\mathrm{N}$ content in grain and straw presented in table 4.0. The content of $\mathrm{N}$ increased with the increasing dose of RDN from 75 to $100 \%$. It was further increased with addition of VC or FYM in combinations of 75 or $100 \% \mathrm{RDN}$. The maximum $\mathrm{N}$ content was recorded with $\mathrm{T}_{14}(100 \% \quad \mathrm{RDN}+25 \% \quad \mathrm{~N}$ $\mathrm{VC}+\mathrm{S}+\mathrm{Zn}+\mathrm{Az}+\mathrm{PSB}$ ) but found on par with $\mathrm{T}_{13} \quad(100 \% \quad \mathrm{RDN}+25 \% \quad \mathrm{~N}-\mathrm{FYM}+\mathrm{S}+\mathrm{Zn}+$ $\mathrm{Az}+\mathrm{PSB}), \mathrm{T}_{6}(75 \% \mathrm{RDN}+25 \% \mathrm{~N}-\mathrm{VC}+\mathrm{S}+\mathrm{Zn}+$ $\mathrm{Az}+\mathrm{PSB})$ and $\mathrm{T}_{5}(75 \% \mathrm{RDN}+25 \% \mathrm{~N}-\mathrm{FYM}+$ $\mathrm{S}+\mathrm{Zn}+\mathrm{Az}+\mathrm{PSB})$. It is evident from table that the addition of FYM or VC with or without $\mathrm{S}+$ $\mathrm{Zn}+$ Azotobacter +PSB further increased the concentration of $\mathrm{N}$ in wheat. It may be ascribed that organic manure released nutrients following decomposition and mineralization that would have increased the availability of plant nutrient Yadav et al., (2018). The combined application of chemical fertilizers along with organic sources stimulates the concentration of nutrients and because of stimulated microbes flush and improved root growth due to congenial soil physical, chemical and biological condition. Apart from $\mathrm{N}$ fixing biofertilizers enhances the soil $\mathrm{N}$ and PSB produces the organic acids which may partly be responsible for quick release of nutrients resulted into more content of nutrients in grain as well as straw (Kumar and Dhar, 2010; Meena et al., 2014).

\section{Phosphorus content and uptake}

The data of $\mathrm{P}$ content presented in table 4.0 The highest $\mathrm{P}$ content was recorded with $\mathrm{T}_{14}$ $(100 \% \quad \mathrm{RDN}+25 \% \quad \mathrm{~N}-\mathrm{VC}+\mathrm{S}+\mathrm{Zn}+\mathrm{Az}+\mathrm{PSB})$ however, remained statistically at par with the treatments of $\mathrm{T}_{13}(100 \% \mathrm{RDN}+25 \% \mathrm{~N}-\mathrm{FYM}+$ $\mathrm{S}+\mathrm{Zn}+\mathrm{Az}+\mathrm{PSB}), \mathrm{T}_{10}(100 \% \mathrm{RDN}+\mathrm{S}+\mathrm{Zn}+\mathrm{Az}$ $+\mathrm{PSB}), \mathrm{T}_{6}(75 \% \mathrm{RDN}+25 \% \quad \mathrm{~N}-\mathrm{VC}+\mathrm{S}+\mathrm{Zn}+$ $\mathrm{Az}+\mathrm{PSB})$ and $\mathrm{T}_{5}(100 \% \mathrm{RDN}+25 \% \mathrm{~N}-\mathrm{FYM}+$ $\mathrm{S}+\mathrm{Zn}+\mathrm{Az}+\mathrm{PSB}$ ) during both the years and on pooled mean basis. The $\mathrm{P}$ content increased significantly when S, Zn and Azotobacter and PSB applied individually or in combination with 100\% RDN in maize, however, it remained statistically at par with each other. With regard to $75 \%$ RDN combinations, $\mathrm{T}_{6}$ $(75 \% \quad \mathrm{RDN}+25 \% \quad \mathrm{~N}-\mathrm{VC}+\mathrm{S}+\mathrm{Zn}+\mathrm{Az}+\mathrm{PSB})$ showed better response in case of $\mathrm{P}$ but showed at par with $\mathrm{T}_{5}$ during both the years. The $\mathrm{N}$ and $\mathrm{P}$ have mutually synergistic effects resulting into growth stimulation and enhanced uptake of both the elements (Sumner et al., 1986). Additionally, decomposition of organic manures, various phenolic and aliphatic acids are produced which solubilize phosphatase and other phosphate bearing minerals and thereby lowers the phosphate fixation and increase its availability (Dotaniya et al., 2014). Incorporation of FYM and PSB along with inorganic $\mathrm{P}$ increase the availability of $\mathrm{P}$ to crop and mineralization of organic $\mathrm{P}$ due to microbial action and enhanced mobility of P (Sharma et al., 2013; Dwivedi et al., 2007 and Bahadur et al., 2012). 
Table.1 Effect of different treatments on $\mathrm{N}$ and $\mathrm{P}$ content in grain stover of maize

\begin{tabular}{|c|c|c|c|c|c|c|c|c|}
\hline \multirow[t]{2}{*}{ Treatments combinations } & \multicolumn{2}{|c|}{$\begin{array}{l}\text { N content } \\
\text { (\%) Grain }\end{array}$} & \multicolumn{2}{|c|}{$\begin{array}{l}\text { N content }(\%) \\
\text { Stover }\end{array}$} & \multicolumn{2}{|c|}{$\begin{array}{l}\text { P content }(\%) \\
\text { Grain }\end{array}$} & \multicolumn{2}{|c|}{$\begin{array}{l}\text { P content }(\%) \\
\text { Stover }\end{array}$} \\
\hline & $\begin{array}{c}2018- \\
19\end{array}$ & $\begin{array}{c}2019 \\
-20\end{array}$ & $\begin{array}{c}2018- \\
19\end{array}$ & $\begin{array}{c}2019- \\
20\end{array}$ & 2018-19 & 2019-20 & $\begin{array}{l}2018- \\
19\end{array}$ & 2019-20 \\
\hline$T_{1}$. Control & 1.31 & 1.30 & 0.50 & 0.48 & 0.28 & 0.27 & 0.09 & 0.09 \\
\hline $\mathrm{T}_{2 .} .75 \% \mathrm{RDN}$ & 1.36 & 1.38 & 0.53 & 0.56 & 0.31 & 0.33 & 0.13 & 0.12 \\
\hline $\begin{array}{c}\text { T }_{3 .} \text {.75\% RDN+25\% N- } \\
\text { FYM }\end{array}$ & 1.39 & 1.42 & 0.55 & 0.58 & 0.33 & 0.36 & 0.16 & 0.15 \\
\hline $\mathrm{T}_{4} .75 \% \mathrm{RDN}+25 \% \mathrm{~N}-\mathrm{VC}$ & 1.41 & 1.44 & 0.56 & 0.59 & 0.34 & 0.37 & 0.17 & 0.16 \\
\hline $\begin{array}{l}\mathrm{T}_{5 .} .75 \% \mathrm{RDN}+25 \% \mathrm{~N}- \\
\mathrm{FYM}+\mathrm{S}+\mathrm{Zn}+\mathrm{Az} .+\mathrm{PSB}\end{array}$ & 1.51 & 1.54 & 0.65 & 0.68 & 0.41 & 0.45 & 0.26 & 0.24 \\
\hline $\begin{array}{l}\mathrm{T}_{6 .} .75 \% \mathrm{RDN}+25 \% \mathrm{~N}- \\
\mathrm{VC}+\mathrm{S}+\mathrm{Zn}+\mathrm{Az} .+\mathrm{PSB}\end{array}$ & 1.53 & 1.56 & 0.67 & 0.70 & 0.42 & 0.46 & 0.28 & 0.26 \\
\hline $\mathrm{T}_{7 .} .100 \% \mathrm{RDN}$ & 1.42 & 1.45 & 0.57 & 0.60 & 0.35 & 0.38 & 0.18 & 0.17 \\
\hline $\mathrm{T}_{8 .} .100 \% \mathrm{RDN}+\mathrm{S}$ & 1.47 & 1.50 & 0.61 & 0.64 & 0.37 & 0.40 & 0.19 & 0.18 \\
\hline$T_{9 .} .100 \%$ RDN+S+Zn & 1.49 & 1.52 & 0.63 & 0.66 & 0.39 & 0.42 & 0.22 & 0.21 \\
\hline $\begin{array}{c}T_{10} \\
100 \% \mathrm{RDN}+\mathrm{S}+\mathrm{Zn}+\mathrm{Az} .+\mathrm{PSB}\end{array}$ & 1.55 & 1.58 & 0.68 & 0.71 & 0.43 & 0.46 & 0.29 & 0.27 \\
\hline $\begin{array}{c}\mathrm{T}_{11 .} \mathbf{1 0 0 \%} \mathrm{RDN}+25 \% \mathrm{~N}- \\
\text { FYM }\end{array}$ & 1.46 & 1.49 & 0.60 & 0.63 & 0.38 & 0.41 & 0.21 & 0.20 \\
\hline $\mathrm{T}_{12 .} \cdot 100 \% \mathrm{RDN}+25 \%-\mathrm{VC}$ & 1.48 & 1.51 & 0.62 & 0.65 & 0.39 & 0.42 & 0.22 & 0.21 \\
\hline $\begin{array}{c}\text { T}_{13 .} 100 \% \mathrm{RDN}+25 \% \mathrm{~N}- \\
\text { FYM+S+Zn+Az.+PSB }\end{array}$ & 1.56 & 1.59 & 0.69 & 0.72 & 0.44 & 0.48 & 0.31 & 0.29 \\
\hline $\begin{array}{l}\mathrm{T}_{14 .} \mathbf{1 0 0 \%} \mathrm{RDN}+\mathbf{2 5 \%} \mathrm{N}- \\
\mathrm{VC}+\mathrm{S}+\mathrm{Zn}+\mathrm{Az}+\mathrm{PSB}\end{array}$ & 1.58 & 1.61 & 0.71 & 0.74 & 0.46 & 0.50 & 0.32 & 0.30 \\
\hline SE(m) & 0.026 & 0.032 & 0.015 & 0.023 & 0.015 & 0.019 & 0.019 & 0.012 \\
\hline CD $(5 \%)$ & 0.067 & 0.090 & 0.044 & 0.067 & 0.045 & 0.054 & 0.054 & 0.034 \\
\hline
\end{tabular}


Table.2 Effect of treatment on $\mathrm{K}$ and $\mathrm{S}$ content in grain and stover of maize

\begin{tabular}{|c|c|c|c|c|c|c|c|c|}
\hline \multirow[t]{2}{*}{ Treatments combinations } & \multicolumn{2}{|c|}{$\begin{array}{l}\text { K content } \\
\text { (\%) Grain }\end{array}$} & \multicolumn{2}{|c|}{$\begin{array}{l}\text { K content (\%) } \\
\text { Stover }\end{array}$} & \multicolumn{2}{|c|}{$\begin{array}{l}\text { S content (\%) } \\
\text { Grain }\end{array}$} & \multicolumn{2}{|c|}{ S content (\%) Stover } \\
\hline & $\begin{array}{c}2018 \\
-19\end{array}$ & $\begin{array}{c}2019- \\
20\end{array}$ & $\begin{array}{c}2018- \\
19\end{array}$ & $\begin{array}{c}2019- \\
20\end{array}$ & 2018-19 & 2019-20 & 2018-19 & 2019-20 \\
\hline $\mathrm{T}_{1}$. Control & 0.36 & 0.34 & 1.08 & 1.07 & 0.06 & 0.05 & 0.06 & 0.07 \\
\hline $\mathrm{T}_{2 .} . \mathbf{7 5 \%} \mathrm{RDN}$ & 0.39 & 0.42 & 1.1 & 1.13 & 0.07 & 0.09 & 0.12 & 0.11 \\
\hline $\mathrm{T}_{3 .} .75 \% \mathrm{RDN}+25 \% \mathrm{~N}-\mathrm{FYM}$ & 0.41 & 0.44 & 1.12 & 1.15 & 0.09 & 0.12 & 0.15 & 0.13 \\
\hline $\mathrm{T}_{4 .} .75 \% \mathrm{RDN}+25 \% \mathrm{~N}-\mathrm{VC}$ & 0.43 & 0.46 & 1.13 & 1.16 & 0.10 & 0.13 & 0.16 & 0.14 \\
\hline $\begin{array}{c}\mathrm{T}_{5 .} .75 \% \mathrm{RDN}+25 \% \mathrm{~N}- \\
\text { FYM+S+Zn+Az.+PSB }\end{array}$ & 0.51 & 0.54 & 1.22 & 1.25 & 0.20 & 0.24 & 0.24 & 0.22 \\
\hline $\begin{array}{l}\mathrm{T}_{6 .} .75 \% \mathrm{RDN}+25 \% \mathrm{~N}- \\
\mathrm{VC}+\mathrm{S}+\mathrm{Zn}+\mathrm{Az} .+\mathrm{PSB}\end{array}$ & 0.53 & 0.56 & 1.24 & 1.27 & 0.22 & 0.26 & 0.25 & 0.23 \\
\hline $\mathrm{T}_{7.100 \% \mathrm{RDN}}$ & 0.44 & 0.47 & 1.14 & 1.17 & 0.11 & 0.13 & 0.16 & 0.15 \\
\hline $\mathrm{T}_{8 .} .100 \% \mathrm{RDN}+\mathrm{S}$ & 0.46 & 0.49 & 1.16 & 1.19 & 0.15 & 0.18 & 0.19 & 0.17 \\
\hline $\mathrm{T}_{9 .} 100 \% \mathrm{RDN}+\mathrm{S}+\mathrm{Zn}$ & 0.49 & 0.52 & 1.20 & 1.23 & 0.18 & 0.21 & 0.21 & 0.19 \\
\hline $\begin{array}{c}T_{10} \\
100 \% \mathrm{RDN}+\mathrm{S}+\mathrm{Zn}+\mathrm{Az} .+\mathrm{PSB}\end{array}$ & 0.54 & 0.57 & 1.25 & 1.28 & 0.23 & 0.27 & 0.26 & 0.24 \\
\hline $\begin{array}{c}\mathrm{T}_{11 .} \text { 100\% RDN+25\%N - } \\
\text { FYM }\end{array}$ & 0.47 & 0.5 & 1.18 & 1.21 & 0.15 & 0.18 & 0.2 & 0.18 \\
\hline $\mathrm{T}_{12 .} .100 \% \mathrm{RDN}+25 \%-\mathrm{VC}$ & 0.48 & 0.51 & 1.19 & 1.22 & 0.16 & 0.19 & 0.21 & 0.19 \\
\hline $\begin{array}{c}T_{13 .} .100 \% \mathrm{RDN}+25 \% \mathrm{~N}- \\
\text { FYM+S+Zn+Az.+PSB }\end{array}$ & 0.55 & 0.58 & 1.26 & 1.29 & 0.24 & 0.28 & 0.29 & 0.27 \\
\hline $\begin{array}{c}\mathrm{T}_{14 .} \mathbf{1 0 0 \%} \mathrm{RDN}+\mathbf{2 5 \%} \mathrm{N}- \\
\mathrm{VC}+\mathrm{S}+\mathrm{Zn}+\mathrm{Az} .+\mathrm{PSB}\end{array}$ & 0.56 & 0.60 & 1.28 & 1.31 & 0.25 & 0.29 & 0.30 & 0.28 \\
\hline $\mathbf{S E}(\mathbf{m})$ & $\begin{array}{c}0.02 \\
3\end{array}$ & 0.028 & 0.031 & 0.037 & 0.012 & 0.015 & 0.019 & 0.012 \\
\hline $\mathrm{CD}(5 \%)$ & $\begin{array}{c}0.06 \\
7\end{array}$ & 0.081 & 0.099 & 0.122 & 0.036 & 0.045 & 0.054 & 0.034 \\
\hline
\end{tabular}


Table.3 Effect of treatments in $\mathrm{Zn}$ content of grain and stover of maize

\begin{tabular}{|c|c|c|c|c|}
\hline \multirow[t]{2}{*}{ Treatments combinations } & \multicolumn{2}{|c|}{ Zn content Grain (mg kg- } & \multicolumn{2}{|c|}{$\begin{array}{c}\text { Zn content Stover ( } \mathrm{mg} \\
\mathrm{kg}^{-1} \text { ) }\end{array}$} \\
\hline & 2018-19 & 2019-20 & 2018-19 & 2019-20 \\
\hline$T_{1}$. Control & 18.93 & 18.89 & 35.73 & 35.68 \\
\hline $\mathrm{T}_{2} . \mathbf{7 5} \% \mathrm{RDN}$ & 19.36 & 19.40 & 36.21 & 36.25 \\
\hline $\mathrm{T}_{3 .} \mathbf{7 5} \% \mathrm{RDN}+25 \% \mathrm{~N}-\mathrm{FYM}$ & 20.45 & 20.49 & 37.33 & 37.36 \\
\hline $\mathrm{T}_{4} .75 \% \mathrm{RDN}+25 \% \mathrm{~N}-\mathrm{VC}$ & 20.66 & 20.70 & 37.56 & 37.60 \\
\hline $\begin{array}{c}\mathrm{T}_{5 .} .75 \% \mathrm{RDN}+25 \% \mathrm{~N}- \\
\mathrm{FYM}+\mathrm{S}+\mathrm{Zn}+\mathrm{Az} .+\mathrm{PSB}\end{array}$ & 23.53 & 23.58 & 40.68 & 40.73 \\
\hline $\mathrm{T}_{6 .} .75 \% \mathrm{RDN}+25 \% \mathrm{~N}-\mathrm{VC}+\mathrm{S}+\mathrm{Zn}+\mathrm{Az} .+\mathrm{PSB}$ & 23.78 & 23.83 & 40.96 & 41.02 \\
\hline $\mathrm{T}_{7 .} .100 \% \mathrm{RDN}$ & 20.83 & 20.87 & 37.78 & 37.82 \\
\hline $\mathrm{T}_{8 .}$ 100\%RDN+S & 20.95 & 20.99 & 37.93 & 37.97 \\
\hline$T_{9 .} 100 \% \mathrm{RDN}+\mathrm{S}+\mathrm{Zn}$ & 22.06 & 22.10 & 39.15 & 39.19 \\
\hline$T_{10} .100 \% \mathrm{RDN}+\mathrm{S}+\mathrm{Zn}+\mathrm{Az} .+\mathrm{PSB}$ & 23.93 & 23.97 & 41.15 & 41.20 \\
\hline 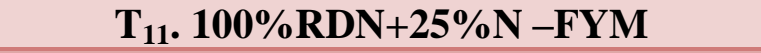 & 21.52 & 21.56 & 38.55 & 38.60 \\
\hline $\mathrm{T}_{12 .} \cdot 100 \% \mathrm{RDN}+25 \%-\mathrm{VC}$ & 21.68 & 21.72 & 38.73 & 38.77 \\
\hline $\begin{array}{c}\mathrm{T}_{13.13} 100 \% \mathrm{RDN}+25 \% \mathrm{~N}- \\
\text { FYM+S+Zn+Az.+PSB }\end{array}$ & 24.03 & 24.08 & 41.26 & 41.11 \\
\hline $\begin{array}{l}\mathrm{T}_{14 .} \mathbf{1 0 0 \%} \mathrm{RDN}+25 \% \mathrm{~N}- \\
\mathrm{VC}+\mathrm{S}+\mathrm{Zn}+\mathrm{Az} .+\mathrm{PSB}\end{array}$ & 24.20 & 24.26 & 41.50 & 41.55 \\
\hline SE(m) & 0.19 & 0.22 & 0.27 & 0.31 \\
\hline $\mathrm{CD}(5 \%)$ & 0.54 & 0.63 & 0.78 & 0.90 \\
\hline
\end{tabular}


Table.4 Effect of different treatments on $\mathrm{N}$ and $\mathrm{P}$ content in grain stover of maize

\begin{tabular}{|c|c|c|c|c|c|c|c|c|}
\hline \multirow[t]{2}{*}{ Treatments combinations } & \multicolumn{2}{|c|}{$\begin{array}{l}\text { N content } \\
\text { (\%) Grain }\end{array}$} & \multicolumn{2}{|c|}{$\begin{array}{l}\text { N content }(\%) \\
\text { Stover }\end{array}$} & \multicolumn{2}{|c|}{$\begin{array}{l}\text { P content }(\%) \\
\text { Grain }\end{array}$} & \multicolumn{2}{|c|}{$\begin{array}{l}\text { P content }(\%) \\
\text { Stover }\end{array}$} \\
\hline & $\begin{array}{c}2018- \\
19\end{array}$ & $\begin{array}{c}2019 \\
-20\end{array}$ & $\begin{array}{c}2018- \\
19\end{array}$ & $\begin{array}{c}2019- \\
20\end{array}$ & 2018-19 & 2019-20 & $\begin{array}{l}2018- \\
19\end{array}$ & 2019-20 \\
\hline$T_{1}$. Control & 1.72 & 1.74 & 0.41 & 0.43 & 0.24 & 0.26 & 0.07 & 0.06 \\
\hline $\mathrm{T}_{2 .} .75 \% \mathrm{RDN}$ & 1.75 & 1.78 & 0.46 & 0.49 & 0.26 & 0.29 & 0.11 & 0.10 \\
\hline $\begin{array}{c}\text { T }_{3 .} \text { 75\% } \\
\text { RDN+25\% N- } \\
\text { FYM }\end{array}$ & 1.79 & 1.81 & 0.5 & 0.53 & 0.29 & 0.32 & 0.14 & 0.13 \\
\hline $\mathrm{T}_{4} .75 \% \mathrm{RDN}+25 \% \mathrm{~N}-\mathrm{VC}$ & 1.80 & 1.83 & 0.51 & 0.54 & 0.30 & 0.33 & 0.15 & 0.14 \\
\hline $\begin{array}{l}\mathrm{T}_{5 .} .75 \% \mathrm{RDN}+25 \% \mathrm{~N}- \\
\mathrm{FYM}+\mathrm{S}+\mathrm{Zn}+\mathrm{Az} .+\mathrm{PSB}\end{array}$ & 1.90 & 1.94 & 0.60 & 0.64 & 0.38 & 0.42 & 0.23 & 0.22 \\
\hline $\begin{array}{l}\mathrm{T}_{6 .} .75 \% \mathrm{RDN}+25 \% \mathrm{~N}- \\
\mathrm{VC}+\mathrm{S}+\mathrm{Zn}+\mathrm{Az} .+\mathrm{PSB}\end{array}$ & 1.91 & 1.95 & 0.61 & 0.65 & 0.39 & 0.43 & 0.24 & 0.23 \\
\hline $\mathrm{T}_{7 .} .100 \% \mathrm{RDN}$ & 1.77 & 1.8 & 0.48 & 0.51 & 0.28 & 0.31 & 0.13 & 0.12 \\
\hline $\mathrm{T}_{8 .} .100 \% \mathrm{RDN}+\mathrm{S}$ & 1.81 & 1.84 & 0.52 & 0.55 & 0.31 & 0.34 & 0.16 & 0.15 \\
\hline$T_{9 .} .100 \%$ RDN+S+Zn & 1.83 & 1.86 & 0.54 & 0.57 & 0.32 & 0.35 & 0.17 & 0.16 \\
\hline $\begin{array}{c}T_{10} \\
100 \% \mathrm{RDN}+\mathrm{S}+\mathrm{Zn}+\mathrm{Az} .+\mathrm{PSB}\end{array}$ & 1.87 & 1.91 & 0.57 & 0.60 & 0.37 & 0.41 & 0.22 & 0.21 \\
\hline $\begin{array}{c}\mathrm{T}_{11 .} \mathbf{1 0 0 \%} \mathrm{RDN}+25 \% \mathrm{~N}- \\
\text { FYM }\end{array}$ & 1.84 & 1.87 & 0.55 & 0.58 & 0.34 & 0.37 & 0.19 & 0.18 \\
\hline $\mathrm{T}_{12 .} \cdot 100 \% \mathrm{RDN}+25 \%-\mathrm{VC}$ & 1.85 & 1.88 & 0.56 & 0.59 & 0.35 & 0.38 & 0.20 & 0.19 \\
\hline $\begin{array}{c}\text { T}_{13 .} 100 \% \mathrm{RDN}+25 \% \mathrm{~N}- \\
\text { FYM+S+Zn+Az.+PSB }\end{array}$ & 1.93 & 1.97 & 0.62 & 0.66 & 0.40 & 0.44 & 0.25 & 0.24 \\
\hline $\begin{array}{l}\mathrm{T}_{14 .} \mathbf{1 0 0 \%} \mathrm{RDN}+\mathbf{2 5 \%} \mathrm{N}- \\
\mathrm{VC}+\mathrm{S}+\mathrm{Zn}+\mathrm{Az}+\mathrm{PSB}\end{array}$ & 1.94 & 1.98 & 0.63 & 0.67 & 0.41 & 0.45 & 0.26 & 0.25 \\
\hline SE(m) & 0.018 & 0.026 & 0.017 & 0.020 & 0.015 & 0.020 & 0.018 & 0.012 \\
\hline CD $(5 \%)$ & 0.054 & 0.067 & 0.050 & 0.058 & 0.044 & 0.058 & 0.053 & 0.034 \\
\hline
\end{tabular}


Table.5 Effect of treatment on $\mathrm{K}$ and $\mathrm{S}$ content in grain and stover of maize

\begin{tabular}{|c|c|c|c|c|c|c|c|c|}
\hline \multirow[t]{2}{*}{ Treatments combinations } & \multicolumn{2}{|c|}{$\begin{array}{l}\mathrm{K} \text { content (\%) } \\
\text { Grain }\end{array}$} & \multicolumn{2}{|c|}{$\begin{array}{l}\text { K content (\%) } \\
\text { Stover }\end{array}$} & \multicolumn{2}{|c|}{$\begin{array}{l}\text { S content (\%) } \\
\text { Grain }\end{array}$} & \multicolumn{2}{|c|}{ S content (\%) Stover } \\
\hline & $\begin{array}{c}2018 \\
19\end{array}$ & $\begin{array}{c}2019- \\
20\end{array}$ & $\begin{array}{c}2018 \\
19\end{array}$ & $\begin{array}{c}2019- \\
20\end{array}$ & $\begin{array}{c}\text { 2018- } \\
19\end{array}$ & 2019-20 & 2018-19 & 2019-20 \\
\hline$T_{1}$. Control & 0.46 & 0.49 & 1.18 & 1.21 & 0.11 & 0.13 & 0.13 & 0.12 \\
\hline $\mathrm{T}_{2.75 \% \mathrm{RDN}}$ & 0.48 & 0.51 & 1.21 & 1.24 & 0.13 & 0.15 & 0.16 & 0.14 \\
\hline $\mathrm{T}_{3 .}$.75\% RDN+25\% N-FYM & 0.51 & 0.54 & 1.24 & 1.27 & 0.16 & 0.19 & 0.19 & 0.18 \\
\hline $\mathrm{T}_{4 .} .75 \% \mathrm{RDN}+25 \% \mathrm{~N}-\mathrm{VC}$ & 0.52 & 0.55 & 1.25 & 1.28 & 0.17 & 0.20 & 0.20 & 0.19 \\
\hline $\begin{array}{l}\mathrm{T}_{5 .} .75 \% \mathrm{RDN}+25 \% \mathrm{~N}- \\
\text { FYM+S+Zn+Az.+PSB }\end{array}$ & 0.60 & 0.64 & 1.34 & 1.38 & 0.27 & 0.31 & 0.30 & 0.28 \\
\hline $\begin{array}{l}\mathrm{T}_{6 .} .75 \% \mathrm{RDN}+25 \% \mathrm{~N}- \\
\mathrm{VC}+\mathrm{S}+\mathrm{Zn}+\mathrm{Az} .+\mathrm{PSB}\end{array}$ & 0.61 & 0.65 & 1.35 & 1.39 & 0.28 & 0.32 & 0.31 & 0.29 \\
\hline $\mathrm{T}_{7} .100 \% \mathrm{RDN}$ & 0.50 & 0.53 & 1.23 & 1.26 & 0.14 & 0.16 & 0.17 & 0.16 \\
\hline $\mathrm{T}_{8 .} .100 \% \mathrm{RDN}+\mathrm{S}$ & 0.53 & 0.56 & 1.26 & 1.29 & 0.20 & 0.23 & 0.23 & 0.22 \\
\hline$T_{9 .} 100 \% \mathrm{RDN}+\mathrm{S}+\mathrm{Zn}$ & 0.54 & 0.57 & 1.28 & 1.31 & 0.21 & 0.24 & 0.24 & 0.23 \\
\hline $\begin{array}{c}T_{10} \\
100 \% \mathrm{RDN}+\mathrm{S}+\mathrm{Zn}+\mathrm{Az} .+\mathrm{PSB}\end{array}$ & 0.59 & 0.62 & 1.33 & 1.37 & 0.26 & 0.30 & 0.28 & 0.28 \\
\hline $\begin{array}{c}\mathrm{T}_{11 .} \cdot 100 \% \text { RDN+25\%N }- \\
\text { FYM }\end{array}$ & 0.56 & 0.59 & 1.30 & 1.33 & 0.23 & 0.26 & 0.25 & 0.24 \\
\hline $\mathrm{T}_{12 .} .100 \% \mathrm{RDN}+25 \%-\mathrm{VC}$ & 0.57 & 0.60 & 1.31 & 1.34 & 0.24 & 0.27 & 0.26 & 0.25 \\
\hline $\begin{array}{l}\mathrm{T}_{13 .} 100 \% \mathrm{RDN}+25 \% \mathrm{~N}- \\
\text { FYM+S+Zn+Az.+PSB }\end{array}$ & 0.63 & 0.67 & 1.37 & 1.41 & 0.29 & 0.33 & 0.32 & 0.30 \\
\hline $\begin{array}{c}T_{14 .} \cdot 100 \% \text { RDN+25\% N- } \\
\text { VC+S+Zn+Az.+PSB }\end{array}$ & 0.64 & 0.68 & 1.38 & 1.42 & 0.30 & 0.34 & 0.33 & 0.31 \\
\hline SE(m) & 0.015 & 0.018 & 0.028 & 0.032 & 0.017 & 0.20 & 0.020 & 0.013 \\
\hline CD (5\%) & 0.045 & 0.054 & 0.081 & 0.094 & 0.049 & 0.058 & 0.059 & 0.037 \\
\hline
\end{tabular}


Table.6 Effect of treatments in $\mathrm{Zn}$ content of grain and stover of maize

\begin{tabular}{|c|c|c|c|c|}
\hline \multirow[t]{2}{*}{ Treatments combinations } & \multicolumn{2}{|c|}{ Zn content $\left(\mathrm{mg} \mathrm{kg}^{-1}\right)$ Grain } & \multicolumn{2}{|c|}{$\begin{array}{c}\text { Zn content }\left(\mathrm{mg} \mathrm{kg}^{-1}\right) \\
\text { Straw }\end{array}$} \\
\hline & 2018-19 & 2019-20 & 2018-19 & 2019-20 \\
\hline$T_{1}$. Control & 37.70 & 37.73 & 8.93 & 8.95 \\
\hline $\mathrm{T}_{2 .} .75 \% \mathrm{RDN}$ & 38.22 & 38.26 & 9.45 & 9.49 \\
\hline $\mathrm{T}_{3 .} .75 \% \mathrm{RDN}+25 \% \mathrm{~N}-\mathrm{FYM}$ & 39.15 & 39.19 & 10.88 & 10.37 \\
\hline $\mathrm{T}_{4} .75 \% \mathrm{RDN}+25 \% \mathrm{~N}-\mathrm{VC}$ & 39.18 & 39.22 & 11.05 & 11.54 \\
\hline $\begin{array}{c}\mathrm{T}_{5 .} .75 \% \mathrm{RDN}+25 \% \mathrm{~N}- \\
\text { FYM+S+Zn+Az.+PSB }\end{array}$ & 41.35 & 41.40 & 13.85 & 13.90 \\
\hline $\mathrm{T}_{6 .} .75 \% \mathrm{RDN}+25 \% \mathrm{~N}-\mathrm{VC}+\mathrm{S}+\mathrm{Zn}+\mathrm{Az} .+\mathrm{PSB}$ & 41.50 & 41.55 & 14.03 & 14.08 \\
\hline $\mathrm{T}_{7 .} .100 \% \mathrm{RDN}$ & 38.66 & 38.70 & 9.88 & 9.92 \\
\hline $\mathrm{T}_{8 .} \mathbf{1 0 0 \% \mathrm { RDN } + \mathrm { S }}$ & 39.45 & 39.49 & 11.82 & 11.86 \\
\hline $\mathrm{T}_{9 .} .100 \% \mathrm{RDN}+\mathrm{S}+\mathrm{Zn}$ & 40.78 & 40.82 & 12.77 & 12.81 \\
\hline$T_{10 .} .100 \% \mathrm{RDN}+\mathrm{S}+\mathrm{Zn}+\mathrm{Az} .+\mathrm{PSB}$ & 41.13 & 41.18 & 13.60 & 13.65 \\
\hline $\mathrm{T}_{11 .} .100 \% \mathrm{RDN}+25 \% \mathrm{~N}-\mathrm{FYM}$ & 40.25 & 40.29 & 12.15 & 12.19 \\
\hline $\mathrm{T}_{12 .} .100 \% \mathrm{RDN}+25 \%-\mathrm{VC}$ & 40.29 & 40.33 & 12.18 & 12.22 \\
\hline $\begin{array}{l}\mathrm{T}_{13.100 \% \mathrm{RDN}+25 \% \mathrm{~N}-} \\
\text { FYM+S+Zn+Az.+PSB }\end{array}$ & 42.03 & 42.08 & 14.60 & 14.65 \\
\hline $\begin{array}{l}T_{14} \cdot 100 \% \mathrm{RDN}+25 \% \mathrm{~N}- \\
\mathrm{VC}+\mathrm{S}+\mathrm{Zn}+\mathrm{Az} .+\mathrm{PSB}\end{array}$ & 42.21 & 42.26 & 14.82 & 14.87 \\
\hline SE $(\mathbf{m})$ & 0.46 & 0.62 & 0.31 & 0.35 \\
\hline CD (5\%) & 1.34 & 1.80 & 0.90 & 1.01 \\
\hline
\end{tabular}

\section{Potassium content}

The highest $\mathrm{K}$ content (grain and straw) was noted with the application of $\mathrm{T}_{14}(100 \%$ $\mathrm{RDN}+25 \% \mathrm{~N}-\mathrm{VC}+\mathrm{S}+\mathrm{Zn}+\mathrm{Az}+\mathrm{PSB})$ however showed at par effect with the treatments of $\mathrm{T}_{13}$ $(100 \% \mathrm{RDN}+25 \% \mathrm{~N}-\mathrm{FYM}+\mathrm{S}+\mathrm{Zn}+\mathrm{Az}+\mathrm{PSB})$, $\mathrm{T}_{6}(75 \% \mathrm{RDN}+25 \% \quad \mathrm{~N}-\mathrm{VC}+\mathrm{S}+\mathrm{Zn}+\mathrm{Az}+\mathrm{PSB})$ $\begin{array}{lllll}\text { and } & \mathrm{T}_{5} & (75 \% & \mathrm{RDN}+25 \% & \mathrm{~N}-\mathrm{FYM}+\mathrm{S}+\end{array}$ $\mathrm{Zn}+\mathrm{Az}+\mathrm{PSB}$ ) but had numerically higher. The $\mathrm{K}$ content and its uptake by grain and straw were influenced significantly with the application of different treatments in maize. In general, the $\mathrm{K}$ content was recorded higher in straw than grain. The results are in close conformity with the findings of (Vasanthy and Kumaraswamy 2000). The increased uptake of $\mathrm{K}$ by wheat may be ascribed to the release of $\mathrm{K}$ from the $\mathrm{K}$-bearing minerals by complexing agents and organic acids produced during decomposition of organic sources (Sharma et al., 2013). Available $\mathrm{K}$ in soil increased with the application of organic manures because of its solubilising action of organic acids produced during FYM decomposition and its higher capacity to hold $\mathrm{K}$ in available form thereby improving $\mathrm{K}$ use efficiency in plants (Vidyavathi et al., 2011).

\section{Sulfur content}

The data regarding $\mathrm{S}$ content have been presented in table 5.0. The application of $S$ in kharif significantly improved the $\mathrm{S}$ content and in grain and straw of wheat as compare to treatments where $\mathrm{S}$ was not applied. The highest $\mathrm{S}$ content was recorded with the treatment of $\mathrm{T}_{14} \quad(100 \% \quad \mathrm{RDN}+25 \% \quad \mathrm{~N}-$ $\mathrm{VC}+\mathrm{S}+\mathrm{Zn}+\mathrm{Az}+\mathrm{PSB})$ which remained statistically on par with $\mathrm{T}_{13}(100 \% \mathrm{RDN}+25 \%$ 
$\mathrm{N}-\mathrm{FYM}+\mathrm{S}+\mathrm{Zn}+\mathrm{Az}+\mathrm{PSB}), \quad \mathrm{T}_{6} \quad(75 \% \quad \mathrm{RDN}+$ $25 \% \mathrm{~N}-\mathrm{VC}+\mathrm{S}+\mathrm{Zn}+\mathrm{Az}+\mathrm{PSB})$ and $\mathrm{T}_{5}(75 \%$ $\mathrm{RDN}+25 \% \quad \mathrm{~N}-\mathrm{FYM}+\mathrm{S}+\mathrm{Zn}+\mathrm{Az}+\mathrm{PSB})$ and significantly superior to rest of the treatments. It was also observed that the application of $S$ in combination with FYM or VC further increased the $\mathrm{S}$ content in grain. The results are in agreement with the findings of Srivastava et al., (2015). The response of wheat to applied $S$ in preceding maize crop may be attributed to an enhanced availability of S in soil Singh and Pandey (2018). The build-up of sulphate $S$ content in soil due to use of FYM either alone or in combination with NPK was reported by Singh et al., (1999) The similar results also reported by Manna et al., (2006).

\section{Zinc content}

The applied $\mathrm{Zn}$ in kharif markedly improved its content in succeeding wheat crop. The maximum $\mathrm{Zn}$ content was recorded with the treatment of $\mathrm{T}_{14} \quad(100 \% \quad \mathrm{RDN}+25 \% \quad \mathrm{~N}$ $\mathrm{VC}+\mathrm{S}+\mathrm{Zn}+\mathrm{Az}+\mathrm{PSB}$ ) which was numerically higher to all the treatments however, remained statistically at par with $\mathrm{T}_{13}(100 \% \mathrm{RDN}+25 \%$ $\mathrm{N}-\mathrm{FYM}+\mathrm{S}+\mathrm{Zn}+\mathrm{Az}+\mathrm{PSB}), \quad \mathrm{T}_{6} \quad(75 \% \quad \mathrm{RDN}+$ $25 \% \mathrm{~N}-\mathrm{VC}+\mathrm{S}+\mathrm{Zn}+\mathrm{Az}+\mathrm{PSB})$ and $\mathrm{T}_{5}(75 \%$ $\mathrm{RDN}+25 \% \mathrm{~N}-\mathrm{FYM}+\mathrm{S}+\mathrm{Zn}+\mathrm{Az}+\mathrm{PSB})$ during both the years of experimentation. These results are in close conformity with the findings of Faujdar and Sharma (2013) and Joshi et al., (2013).

Well decomposed FYM and Vermicompost might have involved in formation of chelates with organic ligands which might have lowered susceptibility to adsorption, fixation and precipitation in the soil and also it was attributed to mineralization of organic manures and consequent release of micronutrients Vidyavathi et al., (2012). The highest concentration of nutrients in maize and wheat were recorded with the application of $\begin{array}{llll}\mathrm{T}_{14} & (100 \% & \mathrm{RDN}+25 \% & \mathrm{~N}-\end{array}$
$\mathrm{VC}+\mathrm{S}+\mathrm{Zn}+\mathrm{Az}+\mathrm{PSB})$ but remained statistically at par with the treatments of $\mathrm{T}_{13}$ where FYM was given instead of VC with respect to all the nutrients including NPKS Zn. It was noticed that the integration of VC/FYM along RDN with or without $\mathrm{S}, \mathrm{Zn}, \mathrm{Az}$ and PSB found optimum from nutrient accumulation point of view. The further addition of $25 \% \mathrm{~N}$ through VC/FYM along $100 \%$ RDN proved beneficial in terms of nutrient concentration in grain and stover of maize and wheat.

\section{References}

Bahadur, Lal, Tiwari, D. D., Mishra, J. and Gupta, B. R. 2012. Effect of integrated nutrient management on yield, microbial population and changes in soil properties under rice-wheat cropping system in sodic soil. Journal of the Indian Society of Soil Science, 60(4): 326- 329.

Balyan J K, Singh, P., Kumpawat, B S., and Jat M L 2008. Effect of organic manure, fertilizer level and biofertilizers on soil nutrients balance in maize (Zea mays L.). Res. on Crops, 9 (2): 308-310.

Blair, N. R., Faulkner, D., Till, A. R., and Poulton, P. R. (2005). Long-term management impacts on soil $\mathrm{C}, \mathrm{N}$ and physical fertility. Soil Till.res., 91: 30-38.

Brar, B. S., Dhillon, N. S. and China, H. S. 2001. Integrated use of farm yard manure and inorganic fertilizers in maize (Zea mays L.). Indian Journal of Agriculture Sciences, 71(9): 605-607.

Badruddin, M., Reynolds, M. P., and Ageeb, O. A. A. (1999). Wheat management in warm environments: Effect of organic and inorganic fertilizers, irrigation frequency and mulching. Agron. J., 91, 975-983.

Brar, B. S., Singh, J., Singh, G., and Kumar, G. (2015). Effects of long term application of inorganic and organic fertilizers on soil organic carbon and physical properties in Maize-Wheat rotation. Agron., (5): 220238.

Dotaniya M L, Datta S C, Biswas D R, Meena H 
M, Kumar K 2014 a. Production of oxalic acid as influenced by the application of organic residue and its effect on phosphorus uptake by wheat (Triticum aestivum L.) in an Inceptisol of north India. Na. Acad. Sci. Lett. 37(5):401-405.

Dwivedi, A. K., Singh, Muneshwar, Kauraw, D. L., Wanjari, R. H. and Chauhan, S. 2007. Impact of fertilizer and manure use for three decades on crop productivity and sustainability, and soil quality under soybean - wheat system on a Vertisol in Central India. Research Bulletin of All India Coordinated Research Project on Long-Term Fertilizer Experiment, Department of Soil Science and Agricultural Chemistry, Jawaharlal Nehru Krishi Vishwa Vidyalaya, Jabalpur (M. P.).

Faujdar, S. R., and Mahendra, S. 2013. Effect of FYM, Biofertilizers and Zinc on yield of Maize and their residual effect on Wheat. J. of Crops and soils, 23 (1): 4152.

Hossain, S. M. A., Kamal, A. M. A., Islam, M. R., and Mannan M. A. (2002). Effects of different levels of chemical and organic fertilizers on growth, yield and protein content of wheat. J. Bio. Sci., 2: 304-306.

Jat, M. L., Sahrawat, Y. S., and Gupta, R. (2011). Conservation agriculture in cereal systems of South Asia: Nutrient management perspectives. Kar. J.Agri. Sci.,24, 100-105.

Joshi E, Nepalia V, Verma A, Singh D. 2013. Effect of integrated nutrient management on growth, productivity and economics of maize (Zea mays L.). Indian Journal of Agronomy, 58 (3):434. 221.

Jackson, M. L.: Soil Chemical Analysis. Prentice Hall of India Pvt. Ltd., New Delhi, pp -498. 1967.

Kuniyal, H. C., Veer Singh, V. Ram, S. and Bhatnagar, A. 2012. Nutrient management on soil health, nutrient uptake and yield of maize under temporary submerged condition in mollisol. J.Madras Agric., 99(7):548-552.

Kumar A. and Shivadhar, 2010. Evaluation of organic and inorganic sources of nutrients in maize (Zea mays) and their residual effect on wheat (Triticum aestivum) under different fertility levels. Indian Journal of Agricultural Sciences, 80 (5): 364-71.

Kundu, S., R. Bhattacharyya, V. Parkash, B. N. Ghosh and Gupta, H. S. (2006). Carbon sequestration and relationship between carbon addition and storage under rainfed soybean-wheat rotation in a sandy loam soil of the India Himalayas. Soil Till. Res., 92: 87-95.

Karki, T. B., Kumar, A. and Gautam, R. C. 2005. Influence of INM on growth, yield, content and uptake of nutrient and soil fertility status in maize (Zea mays L.). Indian Journal of Agricultural Science, 75: 682-685.

Manna, M. C., Swarup, A., Wanjari, R. H., Ravankar, H. N., Mishra, B., Saha, M. N., Singh, Y. V., Sahi, D. K., and Sarap, P. (2005). Long-term effect of fertilizer and manure application on soil organic carbon storage, soil quality and yield sustainability under sub-humid and semiarid tropical India. Field Crops Res., 93, 264-280.

Meena O P, Guar B L, Singh P 2006. Effect of row ratio and fertility levels on productivity, economics and nutrient uptake in maize (Zea mays L.) - soybean (Glycine max) intercropping system. Indian Journal of Agronomy, 51:178-182.

Meena, B. P., Kumar, A., Lal, B., Nishant K. Sinha, P., Tiwari, K., Dotaniya, M. L., Jat, K. N. and Meena, V. D. 2014. Soil microbial, chemical properties and crop productivity as affected by organic manure application in popcorn (Zea mays L. var. everta). African Journal of Microbiology Research, 9(21):1402-1408.

Patil, P. V.; Chalwade, P. B.; Solonke, A. S. and Kulkarni, V. K. 2003. Effect of fly ash and FYM on Physico-chemical properties of vertisol soil and crops, Journal of Soil and Crop. 13 (1):59-64.

Rasool, S., Hamid, S., Kanth, R. H., and Khan, M. H. 2016. Effect of Integrated Nutrient Management on quality, nutrient content 
and uptake of Sweet Corn (Zea mays var. saccharata). American Journal of Experimental Agriculture, 13(6): 1-11.

Rayees A. S. and Wani, B. A. 2017. Yield, nutrient uptake and soil fertility of maize (Zea mays L.) as influenced by varying nutrient management practices under temperate conditions of Kashmir valley, India. Plant Archieve, 17 (1): 75-78.

Steinbach, H. S., Alvarez, R. and Valentz, C. R. 2004. Balance between mineralization and immobization of nitrogen as affected by soil mineral nitrogen level. Agrochemica 48: 204-212.

Singh, G., Somani, L. L., and Totawat, K. L. 1999 a. Scope of supplementing nitrogen requirement of wheat through organic source and Azotobacter. International Journal of Tropical Agriculture, 17: 177182.

Singh, V. and Pandey, M. 2018. Direct effect of sulphur and zinc on productivity, quality and nutrient uptake by pearl millet (Pennisetum glaucum) and their residual effect on succeeding wheat (Triticum aestivum) in pearl millet - wheat crop sequence. Annals of Plant and Soil Research, 20(3): 233-238.

Sharma, G. D., Thakur, R., Som Raj, Kauraw, D. L., and Kulhare, P. S. 2013. Impact of integrated nutrient management on yield, nutrient uptake, protein content of wheat (Triticum aestivum) and soil fertility in a typic haplustert. The Bioscan, 8(4): 11591164.
Sumner, M. E. and Farina, M. P. W. 1986. Phosphorus interactions with other nutrients and lime in field cropping systems. Adv. Soil Sci., 5:201-236.

Singhal, S. K., Sharma, V. K., and Singh, R. D. (2012). Effect of Inorganic \& Biofertilizers (VAM \& PSB) on Yield of Maize and Wheat cropping sequence and soil fertility. Indian J. Agril. Sci. 46(2): 167-172.

Vidyavathi, D. G. S., Babalad, H. B., Hebsur, N. S., Gali, S. K., Patil, S. G. and Alagawadi, A. R. 2012. Nutrient status of soil under different nutrient and crop management practices. Karnataka Journal of Agricultural Sciences, 25 (2): (193-198).

Vasanthi, D. and Kumaraswamy. 2000. Efficacy of vermicompost to improve soil fertility and rice yield. Journal of Indian Society of Soil Science, 47:268-272.

Wagh D S, 2002. Effect of spacing and integrated nutrient management on growth and yield of sweet corn (Zea mays saccharata). M.Sc. (Agri.) Thesis, Mahtama Phule Krishi Vidyapeeth, Rahuri, Dist. Pune.

Wilkinson, S. R., Grunes, D. L. and Sumner, M. E. 1999. Nutrient Interactions in Soil and Plant Nutrition. In Handbook of Soil Science; Sumner, M.E., Ed.; CRC Press: Boca Raton, FL, 89 \pm 112 .

Yadav, K. K. Singh, S. P. Nishant and Kumar, V. 2018. Journal of Experimental Agriculture International, 24(2): 1-9.

\section{How to cite this article:}

Pushpendra Kumar, S. D. Dubey, U. S. Tiwari, R. K. Pandey, Karam Hussain and Singh, R. K. 2021. Effect of INM on Content of NPKS Zn in Grain and Stover of Maize-Wheat Cropping System. Int.J.Curr.Microbiol.App.Sci. 10(06): 750-763.

doi: https://doi.org/10.20546/ijcmas.2021.1006.082 